\title{
Quantitative approach into the heat transfer by convection in microelectronics
}

\author{
by B. Więcek
}

Institute of Electronics, Technical University of Łódž, 18-22 Stefanowskiego str. 90-924 Łódž, Poland

\begin{abstract}
Heat is dissipated to the ambient by convection and radiation in every electronic device. In the long chain of thermal conductors from a heat source (semiconductor) to the ambient (fluid), convection has its significant contribution, especially in steady, long-term conditions. This paper presents 2-D modelling of convective heat dissipation for vertically placed electronic elements with non-uniform temperature distribution. One describes heat removal by convection using the convective transfer coefficient $\alpha$, which depends upon the temperature and the component size. In this paper we evaluate the transfer coefficient by 2-D natural convection simulation.
\end{abstract}

\section{Introduction}

Recent publications indicate a growing interest of thermal analysis in semiconductor power devices and integrated circuits. In the majority of the published works, convection is described by approximated transfer coefficients taken from tables. Many authors assume a uniform distribution of the transfer coefficient, even for large heat removing surfaces [1-2].

In practice, heat transfer by convection is very important in any thermal modelling of microelectronic devices, and has an influence on the accuracy of the entire heat removal model. Although the problem of convection is well known in non microelectronic applications [1-3], it is still not included into the compact thermal analysis, because of high difficulties in getting satisfactory results. There are known analytical [1-2], simplified 1-D [1-2,4] and a 2-D numerical solutions of the Navier-Stokes (N-S) equations [3]. This paper describes 2-D numerical approach of convection modelling for vertical electronic components with a highly non-uniform distribution of the temperature. The main goal of this work is to confirm or update the frequently proposed non-linear characteristics of the convective transfer coefficient derived for the constant temperature of the heat source, and by solving the $1-D$, simplified $N-S$ equations $(1 / 4$ Law), $[1-2,4]$.

$$
\alpha_{c}=\alpha_{0}\left(\frac{\Delta T}{\Delta T_{0}}\right)^{\frac{1}{4}}
$$

where $\alpha_{0}$ denote the transfer coefficient corresponding to temperature difference $\Delta T_{o_{s}}=T-T_{a}$

A long resistor placed in the middle of the ceramic substrate (Fig.1) has been chosen for simulation and experiment. It provides a non-uniform temperature distribution, and measurable difference of temperature on the bottom and top side of the substrate caused by convection.

\section{2-D model for natural convection}

A heat source-hybrid resistor of a few $\mathrm{cm}$ length and of a few tens of $\mu \mathrm{m}$ thickness $\left(h_{\mathrm{r}}\right)$ is placed on the ceramic substrate as shown in Fig. 1. We assume 1-D heat conduction in the substrate because of $h_{s} / h_{r} \gg>1$ and $h_{s} / t_{s}<<1$, where $h_{r}, h_{s}$ and $t_{s}$ are the resistor height, height and thickness of the substrate. The hybrid resistor is placed horizontally in the middle of a vertical ceramic substrate. The length and the height of the resistor are the following: $h_{r}=50 \mu \mathrm{m}$, $\mathrm{L}=30 \mathrm{~mm}$. The 2-D model for heat and mass transfer in the surrounding air is established for an 


\section{http://dx.doi.org/10.21611/qirt.1996.030}

extended region, two times larger than the ceramic substrate height, because of unknown boundary conditions for temperature and velocity on the top of the substrate.

One assumes laminar flow of the fluid, which is valid for $10^{4}<\mathrm{Gr}_{f} \mathrm{Pr}_{\mathrm{f}}<10^{y}$ [2]. For a typical size of microelectronic components e.g.: $h_{s}=3 \mathrm{~cm}$, for temperature difference of maximum $200^{\circ} \mathrm{C}$, and for the following air parameters in temperature $T_{2}=20^{\circ} \mathrm{C}$ : viscosity $v=1.56^{*} 10^{-5} \mathrm{~m}^{2} / \mathrm{s}$, thermal conductivity $\lambda_{\mathrm{a}}=0.026 \mathrm{~W} / \mathrm{Km}$, density $\rho=1.2 \mathrm{~kg} / \mathrm{m}^{3}, c_{\mathrm{a}}=1 \mathrm{~kJ} / \mathrm{kgK}$, we can get: $\mathrm{Gr}_{\mathrm{f}}=7.25^{*} 10^{7}$, $\mathrm{Pr}_{\mathrm{f}}=0.7$, which indicates that in microelectronics, the laminar flow of the air takes place during natural convection.

For steady state 2-D conditions one may consider only one part of the $\mathrm{N}-\mathrm{S}$ equations set, i.e: the equation for vertical direction only, which takes the form $[1,3]$.

$$
u \frac{\partial v}{\partial x}+v \frac{\partial v}{\partial y}=v\left(\frac{\partial^{2} v}{\partial x^{2}}+\frac{\partial^{2} v}{\partial y^{2}}\right)+g \beta\left(T-T_{a}\right)
$$

where: $u, v, \beta$ and $g$ denote the horizontal and vertical components of the velocity, thermal expansion coefficient and the acceleration of the gravity, respectively.

In eqn. (2) the pressure gradient has been replaced by the apparent weight force per unit fluid volume. The second $\mathrm{N}-\mathrm{S}$ equation for the horizontal direction is neglected. The continuity and energy equations have the typical forms:

$$
\begin{aligned}
& \frac{\partial u}{\partial x}+\frac{\partial v}{\partial y}=0 \\
& u \frac{\partial T}{\partial x}+v \frac{\partial T}{\partial y}=a\left(\frac{\partial^{2} T}{\partial x^{2}}+\frac{\partial^{2} T}{\partial y^{2}}\right) \text { where } a=\frac{\lambda_{a}}{\rho c_{a}}
\end{aligned}
$$

The numerical model of heat conduction in the substrate is derived from the energy conservation law, and can be expressed using heat fluxes [5-6]. There are source and sourceless elements in the substrate. For this work, a heat source of one-grid size is assumed. There is no limit for the number of source elements in the model, and the heat source can be easily extended by any number of one-grid source elements. The energy equations for source and sourceless elements take a form:

$$
S_{s} d y \frac{\partial \varphi_{s}(y)}{\partial y}+2 S_{a} \varphi_{a}(y)=\left\{\begin{array}{c}
P_{z} \\
0
\end{array}\right.
$$

where: $\varphi_{s}=-\left.\lambda_{s} \frac{\partial T}{\partial y}\right|_{x=0}$ and $\varphi_{a}=-\left.\lambda_{a} \frac{\partial T}{\partial x}\right|_{x=0}$ are the heat fluxes in the substrate and in the ambient, $S_{s}$ and $S_{a}$ are the corresponding areas where fluxes cross through, $P_{z}[W m]$ denotes the power per length unit dissipated in the hybrid resistor [5].

Finally, the energy equation for heat conduction in the substrate $(x=0)$ can be expressed as:

$$
-d x \lambda_{s} \frac{\partial^{2} T}{\partial y^{2}}-2 \lambda_{a} \frac{\partial T}{\partial x}=\left\{\begin{array}{c}
P_{z} \\
0
\end{array}\right.
$$

where $\lambda_{\mathrm{s}}$ denotes the substrate thermal conductivity and $d x$ is the thickness $\left(d x=t_{s}\right)$.

There are no well defined boundary conditions for both the temperature and the velocity on the top and right sides of the region of laminar fluid flow (Fig.1). The boundary conditions derived from 2-D numerical model for semi-infinite area are used [5]. The solution can be easily obtained by introducing other variables and solving the set of 1-D ordinary equations by the Runge-Kutta method. We assume the same power dissipated for both models with similarly varying temperature on the substrate [5]. 


\section{http://dx.doi.org/10.21611/qirt.1996.030}

\section{Numerical results}

The numerical model presented above has been implemented in C-code, using the GaussCrout Elimination for solving the heat conduction equations in the substrate, and Gauss-Seidel Iteration method for solving the $N-S$ equations. Both procedures run in series, one after the another. The temperature on the substrate obtained by heat conduction model has been used for calculating the temperature and velocity distribution of the air, and so on. A grid of $50 \times 150$ has been chosen.

The properties of the ceramic substrate and of the air are assumed not to depend upon the temperature and are as follows: $v=1.56^{*} 10^{-5} \mathrm{~m}^{2} / \mathrm{s} T_{a}=300 \mathrm{~K}, \lambda_{\mathrm{s}}=20 \mathrm{~W} / \mathrm{Km}, \lambda_{\mathrm{a}}=0.02 \mathrm{~W} / \mathrm{Km}, \mathrm{h}=6 \mathrm{~cm}$, $w=2 \mathrm{~cm}, t_{s}=0.4 \mathrm{~mm}, h_{s}=3 \mathrm{~cm}$. As it was expected the temperature along the substrate and in the surrounding air is highly non-uniform (Fig.2). The vertical component of the fluid velocity has a maximum close to the substrate. This maximum shifts away from the substrate while the warm air moves up to the top of the heat source. Above the substrate, the temperature of the air drops down, and its velocity rise slows down (Fig.3).

The local transfer coefficient (6) defined by the local heat flux and the local temperature is non-linear, and is lower on the top of the substrate (Fig.4). It is because of the development of the thermal boundary layer.

$$
\alpha,(y)=\frac{\lambda_{a} \frac{\partial T(0, y)}{\partial x}}{T(0, y)-T_{a}}
$$

The results of the 2-D model has been compared with 1-D numerical and analytical solutions for finite and infinite vertical plates, especially with results of the heat transfer governed by $1 / 4$ Law, (Fig.5) There are many definitions of convective heat transfer coefficients. For this work we introduce:

$$
\alpha_{\max }=\frac{P_{z}}{2 h_{s}\left(T_{\max }-T_{a}\right)}, \quad \alpha_{\text {meun }}=\overline{\alpha_{,}(y)}
$$

By using the least square method one can fit the exponential functions to 2-D model results for the maximum and mean temperature, and the transfer coefficient. For such a fitting we assume that $\Delta \mathrm{T}_{0}=20^{\circ} \mathrm{C}$.

The results are as follows:

$$
\begin{array}{cc}
P_{z}=P_{0}\left(\frac{\Delta T_{\max }}{\Delta T_{0}}\right)^{n} & \text { where: } P_{0}=5.94, \mathrm{n}=1.12 \\
P_{z}=P_{0}\left(\frac{\Delta T_{\text {mean }}}{\Delta T_{0}}\right)^{n} & \text { where: } P_{0}=9.38, \mathrm{n}=1.09 \\
\alpha_{\text {max }}=\alpha_{0}\left(\frac{\Delta T_{\text {max }}}{\Delta T_{0}}\right)^{n} & \text { where: } \alpha_{0}=5.06, \mathrm{n}=0.11 \\
\alpha_{\text {mean }}=\alpha_{0}\left(\frac{\Delta T_{\text {mean }}}{\Delta T_{0}}\right)^{n} & \text { where: } \alpha_{0}=5.85, \mathrm{n}=0.16
\end{array}
$$

One of the main conclusion of this paper presented in (8) is that the transfer coefficient does not rise so fast with temperature as it has been assumed by previous works $(1 / 4$ Law). The simulations results with $n=0.11 \ldots .0 .16$, for $\alpha \sim T^{n}$. This is because of the non uniform temperature distribution on the substrate obtained by more accurate 2-D calculations. It also results in a more linear relation $P_{z}=f\left(T_{\max }\right)$, (Fig.5).

\section{Experimental results}

We applied thermography to confirm the computational results. The resistor was placed horizontally on the ceramic substrate. The ceramic itself was vertically situated what makes the 
experiment very close to the modelling. All material parameters and dimensions were properly chosen to match the measurement and the modelling. The full confirmation is not possible, because the radiation is neglected in the model. However, the similar temperature distribution in the substrate has been found. The temperature is not symmetrical (Figs. 6-7). Due to convection, the temperature of the upper side of the resistor becomes higher than at the bottom. This result proves the necessity of including the convection in accurate modelling of heat dissipation in microelectronics. The lower maximum temperature in the measurement is caused by radiation. For thermography surveys the hybrid component has been covered with a high emissive thin laver, which increases the amount of energy emitted by radiation. The radiation will be included in the model in the near future.

\section{Conclusions}

This paper presents the 2-D modeling of heat dissipation by natural convention for nonuniform temperature distribution on the substrate. Although only vertically placed hybrid devices have been considered, the found results are useful wherever the temperature is nonuniform. First of all, the convective heat transfer coefficient does not vary so much with the temperature as it was predicted by (1/4 Law). This denotes that the maximum temperature on the substrate is almost linear versus power. 2-D simulations confirm the real need to include the convection into the accurate heat transfer modelling in microelectronics. Quite satisfactory agreement has been found between the modelling and the thermography experiment, although radiation effects should be included in the next works.

\section{Acknowledgements}

Author thanks Prof. G. De Mey from the University of Gent (ELIS), Belgium for his helpful scientific suggestions and inspirations which have been used in this work.

\section{REFERENCES}

[1] Wiśniewski (S.).- Wymiana ciepła. Warsaw, WNT, 1988 [in Polish].

[2] Holman (J.). - Heat Transfer, McGraw-Hill International Book Company, 1981, ISBN 0-07Y66336-X

[3] Rogers (D.). - Laminar Flow Analysis. Cambridge University Press WNT, New York 1992, ISBN 0-521-41152-1.

[4] De Mey (G.), Boone (E.), Nachtergaele (G.), Demolder (S.) and Rottiers (L.) - Non-linear thermal Effects in Hibrid Circuits, Journal on Hybrid Circuits, 1990, vol. pp. 23-26.

[5] Więcek (B.), De Mey (G.). Evaluation of of heat dissipation by convection for VLSI circuits. Proc. TERMINIC'96 Conf., Budapest, Sept.25-27,1996

[6] Więcek (B.). Radiative and convective heat transfer in microelectronics. Proc. QIRT'96. Stuttgart, Sept. 2-5, 1996 


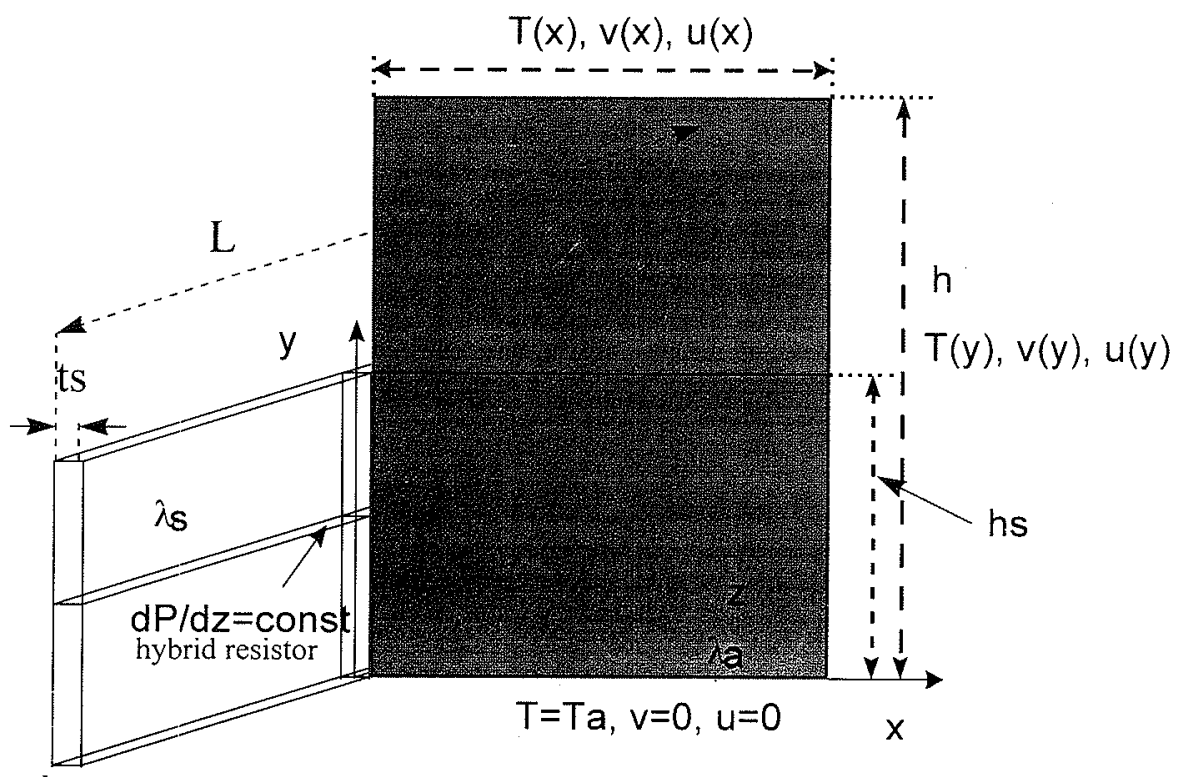

Fig.1. Area for 2-D natural convection model

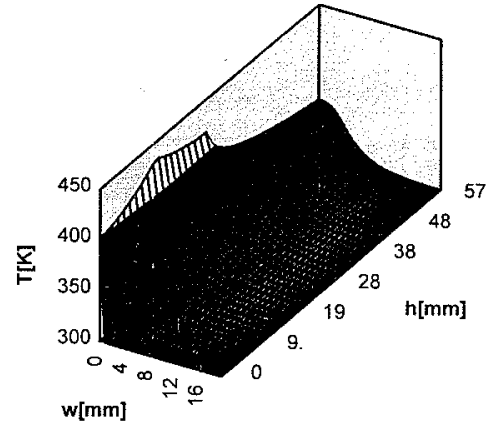

Fig.2. 2-D Temperature distribution, $P_{2}=50 \mathrm{~W} / \mathrm{m}$

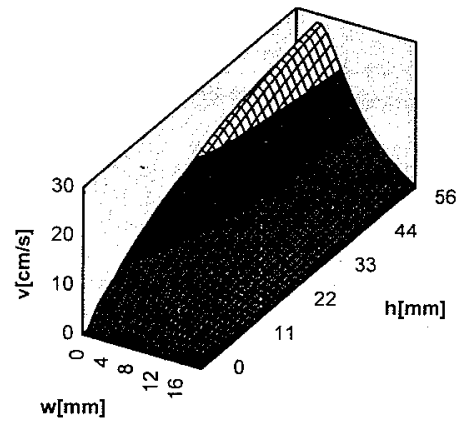

Fig. 3. 2-D vertical velocity distribution, $P_{z}=50 \mathrm{~W} / \mathrm{m}$ 


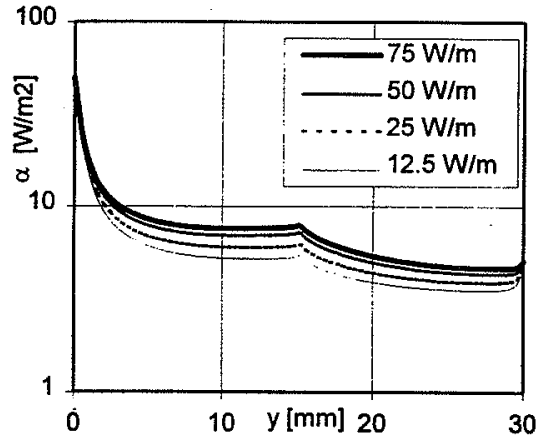

Fig. 4. Local transfer coefficient distribution

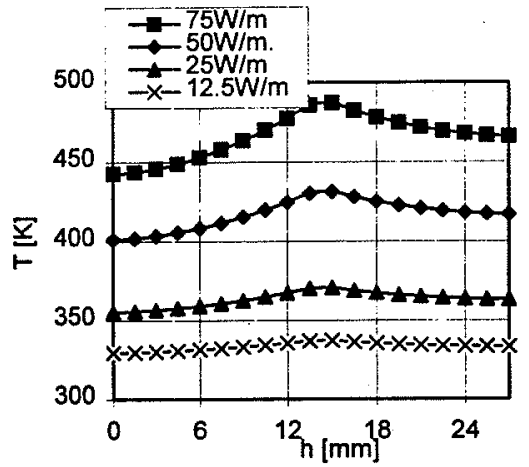

Fig.6. Non symmetrical temperature distribution on the substrate.

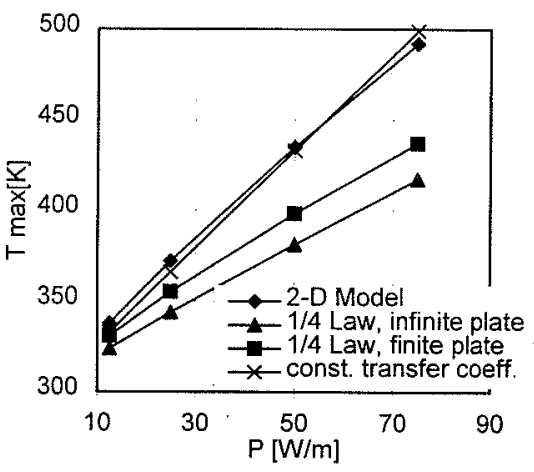

Fig. 5. $T_{\max }$ versus power

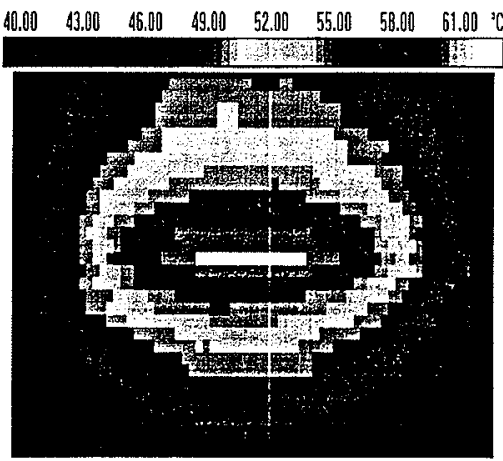

Fig.7. Non symmetrical temperature distribution on the substrate obtained by thermography measurement 